lungen von der heterotypen Teilung bis zur Bildung der Eizelle verlaufen, zweitens konstatierte er, wie viel Abkömmlinge der 4 Kerne, die von den beiden allotypen Mitosen gebildet werden, schließlich in dem 8kernigen Embryosack hereingezogen werden.

Referent möchte das instruktive Schema hier reproduzieren:

\begin{tabular}{|c|c|c|c|c|}
\hline & 5 Teilungen & 4 Teilungen & 3 Teilungen & 2 Teilungen \\
\hline 1 Megaspore & Normaltypus & Codiaeum-Typus & Dicraea-Typus & - \\
\hline 2 Megasporen & - & Scilla-Typus & $\begin{array}{c}\text { Cypripedium - } \\
\text { Typus }\end{array}$ & - \\
\hline 4 Megasporen & 一 & Peperomia-Typus & Lilium-Typus & $\begin{array}{l}\text { Plumbagella }- \\
\text { Typus }^{1} \text { ) }\end{array}$ \\
\hline
\end{tabular}

Damit werden also die 8,4 und 16kernigen Embryosäcke nicht in je eine Kategorie gesteckt, sondern es ist vor allem nach der Vorgeschichte gefragt. Die 8 Kerne des "Normal"- und des "Lilium"-Typus sind so wenig gleichwertig wie die 4 des "Cypripedium". und des "Plumbagella"-Typus usw.

Von jedem Typus beschreibt Verfasser noch eine Anzahl „Modifikationen“. Das wolle man aber im Original nachsehen. Es muß hier genügen, darauf hinzuweisen, daß Verfasser selbst bei Pyrethrum parthenifolium v. aureun eine neue und interessante Form des 16kernigen Embryosacks vom "PeperomiaTypus" aufdeckte.

G. Tischler.

Nawaschin, S. Zellkerndimorphismus bei Galtonia candicans und einigen verwandten Monokotylen. Verhandl. Gesellsch. deutscher Naturf. u. Ärzte. 85. Vers. Wien. II. Teil. p. 629. Leipzig 1913. (vgl. auch die russisch geschriebene Arbeit in Bull. Acad. Imp. Sc. St. Petersburg. Nr. 4 S. 373-385. 5 Fjg. 2 Tab. 3 Diagr. 1912).

Tschernoyarow, M. Über die Chromosomenzahl und besonders beschaffene Chromosomen im Zellkern vou Najas major. Ber. d. d. bot. Ges. Bd. 32. S. 411-416. Taf. 10 (V. M). 1914.

Die Arbeiten von Nawaschin sind dem Ref. nur in Form von kurzen Resumees zugänglich. In der Gesellschaft deutscher Naturforscher und Ärzte gibt der Autor selbst nur eine kurze "Zusammenfassung der Ergebnisse" und die Publikation im Bulletin der Petersburger Akademie ist russisch geschrieben, mithin mir unverständlich geblieben. Allein das Resumé wurde mir seinerzeit von einem Fachgenossen übersetzt. Tschernoyarow, ein Schüler Nawaschins, geht aber auch in seiner Abhandlung auf die Resultate seines Lehrers ein. So können wir uns doch von der Wichtigkeit der hier genannten Arbeiten ein gutes Bild machen.

Darnach existieren im Pflanzenreich "Kerndimorphe zwittrige Arten", die in 2 Rassen existieren, welche sich in ihrem Chromosomenbestand etwas unterscheiden.

Galtonia candicans hat diploid 18 Chromosomen, davon sind aber $2 \mathrm{sehr}$ winzig und diese hängen als "Trabanten" mit 2 anderen zusammen. Bei der Reduktionsteilung fusionieren sie mit ihnen und die Haploidzahl wird so nicht

1) Verfasser kannte Dahlgrens Feststellungen bezüglich dieser Pflanze schon aus einer "Vorl. Mitteil." in Arkiv f. Botan. Bd. 14. 1915. 
9, sondern 8. Die eine Rasse von Galtonia besitzt nun 2 ganz gleiche "Idiochromosomen" in ihrem vegetativen Bestand, während die andere ein größeres und ein kleineres aufweist. (Typus Lygaeus bei den Schmetterlingen). Muscari tenuiflorum besitzt gleichfalls 2 Rassen, aber die eine hat 2, die andere nur ein Idiochromosom, ein „Monosom" (Typus Protenor bei den Schmetterlingen).

Bei Najas marina wies Tschernoyarow endlich ebenfalls 2 nur vegetativ „sichtbare" kleine Idiochromosomen nach, die $\mathrm{zu} 2$ von den 12 größeren im einzelnen genau morphologisch charakterisierten und unterscheidbaren Chromosomen sich als "Trabanten" stellen. Die Haploidzahl ist, wie Guignard seinerzeit richtig angab, wegen der Fusion der Idiochromosomen mit den zugehörigen großen Chromosomen, nur 6. Nach einer "asymmetrischen" Rasse suchte Tschernoyarow aber bisher vergeblich. Vielleicht existiext sie in der Tat nicht und es kommen im mer 2 gleich große Trabanten vor. (Typus Nezara bei den Schmetterlingen).

Der Vergleich mit den bei den Lepidopteren beobachteten Typen ließ zunächst erwarten, daß die Idiochromosomen irgendwie mit der Geschlechtsdeterminierung zusammenhängen. Aber davon zeigte sich bei den untersuchten Pflanzen nichts, sind alles doch $z$ wittrige Arten. Nawaschin meint daher nur, es wäre ,denkbar, daß eine ungerade Chromosomenzahl bezw. überhaupt ein asymmetrischer Chromosomenbestand vielmehr eine Folge oder Spuren der Abstammung der betreffenden Art, wie sonst bekannte Rudimente, darbietet". (In der russischen Abhandlung hatte er noch gemeint: "die Existenz der beiden Rassen kann vielleicht für die Kreuzbefruchtung zweckmäßig sein"). Das klingt nicht sehr befriedigend, aber ehe man überhaupt ein Urteil wird wagen können, dürfte es wichtig sein, festzustellen, ob derartige Kerndimorphismen eine weiter verbreitete Erscheinung repräsentieren.

\section{G. Tischler.}

Pearl, R., 1915. Studies on inbreeding VI. Some further considerations regarding cousin and related kinds of mating. Am. Nat., Vol. 49, $\mathrm{p}, 570-75$.

Eine Ergänzung der Bd. XV, S. 296-98 der Zeitschr. f. ind. Abstammungs- und Vererbungslehre referierten Arbeiten. Zunächst zeigt sich bei den Inzuchtserscheinungen der "single" und „double cousins", daß das Pedigree der früheren Arbeit etwas verändert werden muß, da die Annahme, daß die Hälfte des Inzuchtkoeffizienten der "double cousins" gleich dem der "single cousins" sei, irrig war. Ferner wird ein Pedigree über die Kreuzung Onkel $\times$ Nichte aufgestellt, wobei sich ergibt, daß der Inzuchtkoeffizient der gleiche ist wie bei "single cousins". Aus allen Aufstellungen geht hervor, daß jede ununterbrochene Inzucht, welcher Art die Verwandtschaft auch sei, nach ungefähr 10 Generationen zu einer, bis auf 1 oder $2 \%$, vollkommenen "Konzentration des Blutes" führt. -

Verf. stellt die von Jennings angegebene allgemeine Formel des Inzuchtkoeffizienten der $n$. Generation nun für jeden Verwandtschaftsgrad auf. 